\title{
Metastasen wandern über den JAK2-Stat5- und p38MAPK-Pathway
}

Ein Team der Universität Zürich wies erstmals den Weg nach, den metastasierende Darmkrebszellen von der Blutbahn einschlagen. Dank wirksamer Therapien und guter Früherkennung sterben heute in den Industrienationen nur $10 \%$ der Darmkrebspatienten direkt durch den Primär-

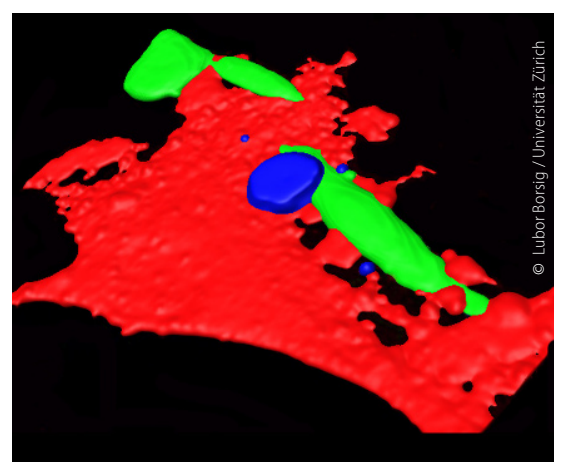

Eine Tumorzelle (grün) hat mit ihrem Chemokin den Rezeptor des Endothels (rot) aktiviert und zwängt sich gerade durch die geöffnete Endothelzelle. Ein Monozyt (blau) hilft ihr dabei. tumor. Häufiger sind die gestreuten Metastasen die Todesursache. Bislang war unbekannt, wieso eine Metastasierung in bestimmte Organe erfolgt und wie die Tochterzellen aus den Gefäßen in Organe eindringen. Nun wies eine Arbeitsgruppe um Lubor Borsig von der Universität Zürich, und Mathias Heikenwälder von der Technische Universität München, nach, dass Krebszellen spezifische Pförtnerrezeptoren auf dem Blutgefäß-Endothel manipulieren. Zum Öffnen der Blutgefäße verwenden sie ein tumoreigenes Chemokin, das Chemokin CCL2. Erhöhte Werte dieses Botenstoffs sind charakteristisch für metastasierende Brust-, Prostata- und Darmkarzinome. Bislang wurden hohe CCL2-Werte vor allem als Hinweis auf ein starkes Tumorwachstum und eine schlechte Krankheitsprognose verstanden. Nun hat sich bei In-vivo- und In-vitro-Experimenten an Labormäusen herausgestellt, dass CCL2 weit mehr ist als ein Indikator für die Aggressivität des Krebs.
„CCL2 aktiviert einen Pförtnerrezeptor auf dem Endothel der Blutgefäße und ermöglicht der Darmkrebszelle so, aus der Blutbahn auszutreten und in anderen Organen zu metastasieren", wird Borsig in einer Mitteilung der Universität Zürich zitiert. Welche Aufgabe dieser Rezeptor als CCR2 bezeichnet - im gesunden Organismus hat, ist nicht bekannt. Borsig vermutet, dass er bei der Immunreaktion des Körpers die Durchlässigkeit der Blutgefäße moduliert. Der neu entdeckte Mechanismus werde damit einen Ansatz für die Entwicklung von Medikamenten gegen Metastasen bei Brust-, Prostata- und Darmkrebs liefern, prognostiziert Borsig. Denkbar sei beispielsweise, die Chemokin-Expression des Tumors zu unterdrücken oder aber den Rezeptor für das Tumor-Chemokin am Endothel zu blockieren, sodass keine Krebszellen mehr aus der Blutbahn in das gesunde Gewebe wandern. „Wenn es gelänge, die Krebszellen am Verlassen der Blutbahnen zu hindern, könnte die Metastasierung direkt am Ursprung bekämpft werden“, hofft der Zürcher Wissenschaftler.

Doris Berger

Wolf MJ et al. Endothelial CCR2 Signaling Induced by Colon Carcinoma Cells Enables Extravasation via the JAK2-Stat5 and p38MAPK Pathway. Cancer Cell. 2012;22(1):91-105.

\section{Therapiearsenal erweitert}

Durch die Monotherapie mit dem Antiandrogen Enzalutamid lebten Patienten mit kastrationsresistentem Prostatakarzinom in der AFFIRM (A Study Evaluating the Efficacy and Safety of the Investigational Drug MDV3100)-Studie signifikant länger im Vergleich zu einer Placebo-Behandlung (18,5 vs. 13,6 Monate).

Fast 1.200 Männer mit kastrationsresistentem Prostatakarzinom nach einer Chemotherapie nahmen an der PhaseIII-Zulassungsstudie teil. Sie erhielten oral entweder $160 \mathrm{mg}$ Enzalutamid/Tag $(n=800)$ oder ein Placebo $(n=399)$. Primärer Endpunkt war das Gesamtüberleben. Im Vergleich zur Placebogruppe lag bei der ersten Interimsanalyse die Sterberate in der Verumgruppe um $37 \%$ niedriger, Grund genug, die Studie vorzeitig zu beenden. 520 Männer waren zu diesem Zeitpunkt bereits gestorben.

Die Überlegenheit des Antiandrogens spiegelt sich auch bei den sekundären Endpunkten wider. Wie Howard I. Scher und Kollegen berichten, war in der Enzalutamid-Gruppe:

- der Patientenanteil, bei dem unter anderem der PSA-Wert um $50 \%$ abnahm signifikant höher (54 vs. $2 \%$ )

- die Zeit bis zum Wiederanstieg des PSA-Wertes signifikant länger (8,3 vs. 3,0 Monate).

- die Zeitspanne bis zur radiologisch nachgewiesenen Krankheitsprogression signifikant länger (8,3 vs. 2,9 Monate)

- die Dauer bis zum ersten SRE (skeletalrelated event) signifikant länger (16,7 vs. 13,3 Monate).
SRE war definiert als Knochenbestrahlung oder -operation, Knochenfraktur, Rückenmarkkompression oder Anpassung der Krebstherapie, um Knochenschmerzen zu lindern.

Den Autoren zufolge hat Enzalutamid keine agonistische Aktivität. Die Krankheitsprogression ließ sich trotz niedriger zirkulierender Androgenspiegel verlangsamen. Häufigste unerwünschte Wirkungen des Antiandrogens waren $\mathrm{Fa}$ tigue, Durchfall und Hitzewallungen. Dosisabhängig kann es bei einer Behandlung mit Dosierungen oberhalb der therapeutischen Dosis zu tonisch-klonischen Muskelkrämpfen kommen. Die Zulassung des Präparates wurde auf der Grundlage der Ergebnisse der AFFIRMStudie in Europa und in den USA beantragt.

Peter Leiner

Scher HI et al. Increased Survival with Enzalutamide in Prostate Cancer After Chemotherapy. N Engl J Med. 2012 [Epub ahead of print] 